\title{
riccafd
}

Revista Iberoamericana de Ciencias de la Actividad Física y el Deporte

\section{PERCEPCIÓN DE LAS BARRERAS Y FACILITADORES PARA LA PRÁCTICA DE ACTIVIDAD FÍSICA DE FUTBOLISTAS DE LA LIGA GENUINE}

\section{PERCEPTION OF THE BARRIERS AND FACILITATORS FOR THE PRACTICE OF PHYSICAL ACTIVITY OF FOOTBALL PLAYERS OF THE GENUINE LEAGUE}

Mendia, U1'; Iturricastillo, A¹; Rodríguez-Negro, J13 Romarate, A1; Yanci, J1

${ }^{1}$ Mendia, U. Departamento de Educación Física y Deportiva, Universidad del País Vasco (UPV/EHU), España, uxuemendia21@gmail.com

${ }^{1}$ Iturricastillo, A. Departamento de Educación Física y Deportiva, Universidad del País Vasco (UPV/EHU), España, aitor.iturricastillo@ehu.eus

${ }^{1}$ Rodríguez-Negro, J. Departamento de Educación Física y Deportiva, Universidad del País Vasco (UPV/EHU), España, josune.rodriguez@ehu.eus

${ }^{1}$ Romarate, A. Departamento de Educación Física y Deportiva, Universidad del País Vasco (UPV/EHU), España, aromarate002@ikasle.ehu.eus

${ }^{1}$ Yanci, J. Departamento de Educación Física y Deportiva, Universidad del País Vasco (UPV/EHU), España,_javier.yanci@ehu.eus

Código UNESCO: 5899. Otras Especialidades Pedagógicas (Educación Física y deporte) Clasificación Consejo de Europa: 4. Educación Física y deporte comparado Correspondencia: Javier Yanci. javier.yanci@ehu.eus

DOI: http://dx.doi.org/10.24310/riccafd.2020.v9i1.8304

\section{RESUMEN}

El presente estudio tiene por objetivo analizar la percepción de las barreras y los facilitadores para la práctica de actividad física (AF) en deportistas con discapacidad. En este estudio participaron 23 personas adultas $(30,35 \pm 10,82$ años) con discapacidad intelectual (DI) y parálisis cerebral (PC) que entrenaban y competían asiduamente en un equipo de futbol de la Liga Genuine. Para analizar las barreras y los facilitadores percibidos, los participantes completaron el cuestionario Motivos y Barreras para la Actividad Física y el Deporte (MBAFD). Los resultados de este estudio muestran que las mayores barreras a la hora de practicar AF son la pereza y el no sentirse a gusto con su cuerpo. Cabe destacar que todas las barreras recibieron una puntuación muy baja $(<0,74 \pm$ 1,18). Respecto a los facilitadores, los más puntuados fueron realizar AF por diversión y para sentirse bien consigo mismo. La mayoría de los ítems sobre los 
facilitadores recibieron una alta puntuación. Atendiendo a los resultados obtenidos, podría ser interesante promover una práctica de AF donde se destaquen las tareas grupales, lúdicas y orientadas a la salud y al bienestar. No obstante, el hecho de que los participantes fueron personas con discapacidad asiduamente practicantes de un deporte de equipo y con una amplia experiencia, ha podido condicionar la baja puntuación de las barreras en la mayoría de los ítems.

palabras clave: práctica motriz, salud, inclusión, deporte adaptado, adhesión, hábitos.

\section{ABSTRACT}

The aim of this study is to analyze the perception of barriers and facilitators for the practice of physical activity (PA) in people with disabilities. This study involved 23 adults ( $30.35 \pm 10.82$ years) with intellectual disability (ID) and cerebral palsy (CP) who trained and competed assiduously in a football team of the Genuine League. To analyze the perceived barriers and facilitators, the participants completed the Motives and Barriers to Physical Activity and Sports questionnaire (MBPAS). The results of this study show that the biggest barriers when practicing PA are laziness and not feeling comfortable with their body. It should be noted that all barriers received a very low score $(<0.74 \pm 1.18)$. Regarding the facilitators, the most punctuated ones were performing PA for fun and to feel good with themselves. Most of the items on the facilitators received a high score. In view of the results obtained, it could be interesting to promote a PA practice where tasks are based in the group, in being playful and oriented to health and well-being. However, the fact that the participants were people with disabilities assiduously practicing a team sport and with broad experience, has been able to condition the low score of the barriers in most of the items.

key words: motor practice, health, inclusión, adapted sport, adherence, habits.

\section{INTRODUCCIÓN}

Anteriores estudios han expuesto que la actividad física (AF) es beneficiosa para la mejora de la salud de la población general ya que, entre otros aspectos, mejora la capacidad cardiovascular ${ }^{1}$, reduce el riesgo de padecer enfermedades del sistema cardiovascular ${ }^{2}$, mejora la función neuromuscular ${ }^{3}$, la estructura ósea y la capacidad funcional ${ }^{4}$. Además de los efectos en la salud física, también se ha descrito que una adecuada práctica de AF mejora el bienestar psicológico ${ }^{5}$. Concretamente en personas con discapacidad, al igual que para la población general, también se ha descrito que la actividad física produce múltiples beneficios en la salud ${ }^{6}$, tanto en el plano físico ${ }^{7,8,9}$, psicológico ${ }^{10,11}$ como también en el plano social1 $1^{2,13,14}$. Teniendo en cuenta los múltiples beneficios que la AF tiene para la salud, parece relevante y necesario que las personas con discapacidad realicen práctica de AF de forma regular. 
Sin embargo, a pesar de los beneficios que produce la AF en personas con discapacidad, se ha observado un menor nivel de práctica de AF en comparación con la población general ${ }^{15}$. Por ejemplo, atendiendo a los resultados observados por Wouters et al. ${ }^{16}$ en un estudio reciente, los niños de entre 2 y 18 años con discapacidad intelectual (DI) participantes en la investigación, no alcanzaron los $60 \mathrm{~min}$ de AF diaria recomendada por la Organización Mundial de la Salud ${ }^{4}$. En la misma línea, en otro estudio realizado con niños con parálisis cerebral $(P C)^{17}$, observaron que la actividad física diaria de este colectivo era inferior a los niveles correspondientes a su edad. Este menor nivel de practica de AF también ha sido constatado en población adulta con discapacidad ${ }^{18,19}$. En un estudio realizado por Gallego et al. ${ }^{18}$ se analizaron los hábitos de AF de mujeres adultas con discapacidad, encontrando que el $44,6 \%$ de las participantes no realizaba ningún tipo de AF. En la misma línea, Draheim et al. ${ }^{19}$ analizaron los niveles de práctica de personas adultas con DI, concluyendo que entre el $47 \%$ y el $51 \%$ de los sujetos eran sedentarios, algunos de ellos por no realizar ningún tipo de AF y otros por el bajo nivel de intensidad de la práctica. Además, según el Centro Nacional de Estadísticas de Salud ${ }^{20}$, un $56 \%$ de los adultos con discapacidad no realiza ninguna AF en su tiempo libre, comparado con un $36 \%$ en personas adultas sin discapacidad. Debido al menor nivel de práctica de AF de las personas con discapacidad, uno de los objetivos de los profesionales de la AF debería ser el aumentar y mejorar los niveles de práctica de AF, no solo de la población general sino también de las personas con discapacidad.

Para poder actuar de forma facilitadora en el aumento de los niveles de práctica de AF de las personas con discapacidad o para mejorar la práctica de aquellos que ya la realizan, es indispensable conocer que aspectos facilitan la práctica y que dificultades perciben los propios implicados ${ }^{21}$. Estudios anteriores han analizado cuales son las barreras y los motivos para la práctica de AF percibidos por personas con discapacidad, concluyendo que las mayores barreras a la hora de realizar AF en personas con DI fueron las dificultades con el transporte y el tiempo necesario para llegar al lugar de entrenamiento ${ }^{22}$. En la misma línea, Verschuren et al. ${ }^{23}$, analizaron que las barreras observadas por las personas con PC fueron la falta de energía y las características físicas del individuo. En cuanto a los facilitadores o motivos para la práctica de AF en personas con DI, destacaron el estar saludable, el aumentar la confianza en uno mismo y el aumentar el entorno social22. En otro estudio realizado con personas con $\mathrm{PC}$, los facilitadores o motivos para la práctica de AF más destacados fueron la relación con los compañeros y con los entrenadores y la actitud positiva de los padres ${ }^{23}$. A pesar de existir estudios en estos colectivos, puede ser necesario conocer las barreras o motivos de práctica en otros contextos sociales y deportivos, ya que el contexto y el entorno social o cultural o el nivel de práctica deportiva habitual puede provocar distintas percepciones de las barreras y motivos de práctica de AF.

Por lo tanto, el objetivo de este estudio fue analizar las barreras y los motivos de práctica de AF percibidas por deportistas con discapacidad (DI y PC) que practicaban asiduamente una modalidad deportiva competitiva de equipo. 


\section{MATERIAL Y METODOS}

\section{Participantes}

En este estudio participaron 23 personas adultas con discapacidad (30,35 \pm 10,82 años, $77,65 \pm 15,96 \mathrm{~kg}, 170,96 \pm 9,39 \mathrm{~cm}$ ) que entrenaban y competían en la Liga Genuine de fútbol, de los cuales 18 participantes tenían DI y 5 tenían PC (21 hombres y 2 mujeres). En la tabla 1 se presenta el número total de participantes y divido por sexo y tipo de discapacidad. Los criterios de inclusión considerados en este estudio fueron tener alguna discapacidad y disponer de licencia federativa oficial en vigor para participar en la Liga Genuine. Se excluyó a quienes, aun cumpliendo los criterios de inclusión, no participaban asiduamente en los entrenamientos y partidos, y a quienes se encontraban lesionados en el momento de llevar a cabo la investigación. Todos los participantes fueron informados de los procedimientos y metodología del estudio antes de cumplimentar el cuestionario y podían tomar la decisión libremente de no participar en el estudio. El estudio siguió las pautas marcadas en la Declaración de Helsinki (2013) y fue aprobado por el Comité de Ética para la Investigación con Seres Humanos (CEISH, código M10_2019_058) de la Universidad del País Vasco (UPV/EHU).

Tabla 1. Participantes del estudio $(n=23)$ atendiendo al sexo y al tipo de discapacidad.

\begin{tabular}{lccc}
\hline & Hombres & Mujeres & Total \\
\hline Discapacidad intelectual & 17 & 1 & 18 \\
Parálisis cerebral & 4 & 1 & 5 \\
\hline
\end{tabular}

\section{Procedimiento}

En el mes de marzo, antes de un entrenamiento del equipo, se pasó el cuestionario Motivos y Barreras para la Actividad Física y el Deporte (MBAFD) a todos los participantes en el estudio, con el fin de conocer la percepción sobre las barreras y los motivos de la práctica de AF de los jugadores. El cuestionario fue cumplimentado por cada participante de manera escrita e individual. En caso de que el participante lo solicitara, los investigadores prestaron aclaraciones a cada una de las cuestiones planteadas en el cuestionario. Antes de completar el cuestionario, todos los participantes recibieron información detallada del procedimiento para rellenarlo de forma adecuada.

\section{Mediciones}

Cuestionario MBAFD: Para identificar las barreras y los motivos percibidos por los participantes a la hora de realizar AF se empleó el cuestionario MBAFD, el cual había sido validado anteriormente por Kostiuk ${ }^{24}$ para población adulta. El cuestionario utilizado estaba dividido en dos bloques con un total de 59 ítems o preguntas. 
El primer bloque hacía referencia a los motivos para la práctica de AF y estaba compuesto por un total de 26 ítems o preguntas. El segundo bloque hacía referencia a las barreras para la práctica de AF y estaba compuesto por 27 ítems. Las respuestas de todos los ítems se recogían en una escala tipo Likert (0-3), donde el valor 0 era "nada" y el valor 3 era "mucho" e incluía también una posible opción de no sabe/no contesta (NS/NC).

\section{Análisis estadístico}

Los resultados se presentan como media \pm desviación típica (DT) de la media. También se realizó un análisis descriptivo complementario calculando las frecuencias y porcentajes de las respuestas aportadas por los participantes en cada ítem o pregunta. El análisis estadístico se realizó con el programa Statistical Package for Social Sciences (SPSS Inc, versión 23,0 Chicago, IL, EE.UU.).

\section{RESULTADOS}

En la tabla 2 se presentan los resultados de las barreras percibidas por los participantes a la hora de realizar AF. Los resultados obtenidos muestran que en todos los ítems referentes a las barreras percibidas la media fue inferior a 1 punto (escala de 0 a 3 ) y que los porcentajes de la respuesta "nada" fueron muy elevados en todos los casos $(60,9$ - 100\%). Los ítems o preguntas que suponían una mayor barrera fueron "no me siento a gusto con mi cuerpo" $(0,74 \pm 1,18)$ y "me da pereza" $(0,70 \pm 1,06)$. Por el contrario, los ítems que suponían una menor barrera fueron "no me siento capaz" $(0,00 \pm 0,00)$ y "los técnicos no son adecua-dos" $(0,00 \pm 0,00)$.

En la tabla 3 se exponen los resultados de los motivos percibidos por los participantes para realizar AF. Los resultados obtenidos en el presente estudio muestran que, en muchos de los ítems, la media de las respuestas fue superior a 2 puntos. Los ítems o preguntas que suponían un motivo mejor puntuado fueron "por diversión" $(2,91 \pm 0,29)$ y "porque haciendo actividad física me siento bien conmigo mism@” $(2,87 \pm 0,46)$. Por el contrario, los ítems que suponían un menor motivo para la práctica de AF fueron "por obligación" $(0,26 \pm 0,69)$, "para compartir actividades con mis hij@s" $(0,13 \pm 0,63)$ y "para recuperarme de una enfermedad o lesión" $(0,35 \pm 0,93)$.

\section{DISCUSIÓN}

El objetivo principal de este estudio fue conocer las barreras y los motivos para la práctica de AF percibidas por deportistas con discapacidad. La principal contribución del presente estudio ha sido el análisis de dichas barreras y motivos en participantes que practicaban asiduamente una modalidad deportiva, ya que la mayoría de trabajos existentes se han realizado con personas sedentarias o no practicantes de AF de forma habitual. Los resultados obtenidos en este estudio 
Tabla 2. Resultados de las barreras percibidas por los participantes para la práctica de actividad física.

\begin{tabular}{|c|c|c|c|c|c|}
\hline Ítem/pregunta & Media $\pm \mathrm{DT}$ & Nada & Algo & Bastante & Mucho \\
\hline Temor a hacerme daño & $0,52 \pm 0,79$ & $14(60,9 \%)$ & $7(30,4 \%)$ & $1(4,3 \%)$ & $1(4,3 \%)$ \\
\hline $\begin{array}{l}\text { Me da vergüenza que me } \\
\text { vean haciendo ejercicio }\end{array}$ & $0,09 \pm 0,29$ & $21(91,3 \%)$ & $2(8,7 \%)$ & $0(0 \%)$ & $0(0 \%)$ \\
\hline $\begin{array}{l}\text { No me siento a gusto con mi } \\
\text { cuerpo }\end{array}$ & $0,74 \pm 1,18$ & $16(69,6 \%)$ & $0(0 \%)$ & $4(17,4 \%)$ & $3(13 \%)$ \\
\hline $\begin{array}{l}\text { Mi empleo actual me lo } \\
\text { impide }\end{array}$ & $0,26 \pm 0,69$ & $19(82,6 \%)$ & $3(13 \%)$ & $0(0 \%)$ & $1(4,3 \%)$ \\
\hline Soy demasiado mayor & $0,26 \pm 0,86$ & $21(91,3 \%)$ & $0(0 \%)$ & $0(0 \%)$ & $2(8,7 \%)$ \\
\hline Estoy cansad@ & $0,61 \pm 1,08$ & $16(69,6 \%)$ & 3 (13\%) & $1(4,3 \%)$ & $3(13 \%)$ \\
\hline Es demasiado caro & $0,22 \pm 0,52$ & $19(82,6 \%)$ & $3(13 \%)$ & $1(4,3 \%)$ & $0(0 \%)$ \\
\hline $\begin{array}{l}\text { Los técnicos no son } \\
\text { adecuados }\end{array}$ & $0,00 \pm 0,00$ & $23(100 \%)$ & $0(0 \%)$ & $0(0 \%)$ & $0(0 \%)$ \\
\hline $\begin{array}{l}\text { Los espacios donde puedo } \\
\text { practicar no son adecuados }\end{array}$ & $0,04 \pm 0,21$ & $22(95,7 \%)$ & $1(4,3 \%)$ & $0(0 \%)$ & $0(0 \%)$ \\
\hline $\begin{array}{l}\text { No hay espacios próximos } \\
\text { donde practicar }\end{array}$ & $0,43 \pm 0,79$ & $16(69,6 \%)$ & $5(21,7 \%)$ & $1(4,3 \%)$ & $1(4,3 \%)$ \\
\hline No me gusta & $0,04 \pm 0,21$ & $22(95,7 \%)$ & $1(4,3 \%)$ & $0(0 \%)$ & $0(0 \%)$ \\
\hline $\begin{array}{l}\text { No soy capaz de generar } \\
\text { tiempo para mi }\end{array}$ & $0,39 \pm 0,89$ & $18(78,3 \%)$ & $3(13 \%)$ & $0(0 \%)$ & $2(8,7 \%)$ \\
\hline Me da pereza & $0,70 \pm 1,06$ & $15(65,2 \%)$ & $2(8,7 \%)$ & $4(17,4 \%)$ & $2(8,7 \%)$ \\
\hline No sé dónde hacer ejercicio & $0,30 \pm 0,76$ & $19(82,6 \%)$ & $2(8,7 \%)$ & $1(4,3 \%)$ & $1(4,3 \%)$ \\
\hline Tengo que cuidar a mis hij@s & $0,04 \pm 0,21$ & $22(95,7 \%)$ & $1(4,3 \%)$ & $0(0 \%)$ & $0(0 \%)$ \\
\hline No me siento capaz & $0,00 \pm 0,00$ & $23(100 \%)$ & $0(0 \%)$ & $0(0 \%)$ & $0(0 \%)$ \\
\hline No estoy en forma & $0,61 \pm 1,12$ & $17(73,9 \%)$ & $1(4,3 \%)$ & $2(8,7 \%)$ & $3(13 \%)$ \\
\hline Dificultades de transporte & $0,13 \pm 0,34$ & $20(87 \%)$ & $3(13 \%)$ & $0(0 \%)$ & $0(0 \%)$ \\
\hline $\begin{array}{l}\text { No tengo con quien hacer } \\
\text { ejercicio }\end{array}$ & $0,30 \pm 0,76$ & $19(82,6 \%)$ & $2(8,7 \%)$ & $1(4,3 \%)$ & $1(4,3 \%)$ \\
\hline $\begin{array}{l}\text { Tengo que ocuparme de las } \\
\text { tareas del hogar }\end{array}$ & $0,57 \pm 0,84$ & $14(60,9 \%)$ & $6(26,1 \%)$ & $2(8,7 \%)$ & $1(4,3 \%)$ \\
\hline Tengo mal estado de salud & $0,35 \pm 0,71$ & $17(73,9 \%)$ & $5(21,7 \%)$ & $0(0 \%)$ & $1(4,3 \%)$ \\
\hline Horarios incompatibles & $0,43 \pm 0,84$ & $17(73,9 \%)$ & $3(13 \%)$ & $2(8,7 \%)$ & $1(4,3 \%)$ \\
\hline No tengo costumbre & $0,52 \pm 1,04$ & $17(73,9 \%)$ & $3(13 \%)$ & $0(0 \%)$ & $3(13 \%)$ \\
\hline $\begin{array}{l}\text { No se oferta la actividad que } \\
\text { me gusta }\end{array}$ & $0,04 \pm 0,21$ & $22(95,7 \%)$ & $1(4,3 \%)$ & $0(0 \%)$ & $0(0 \%)$ \\
\hline $\begin{array}{l}\text { No veo necesario hacer } \\
\text { ejercicio }\end{array}$ & $0,09 \pm 0,42$ & $22(95,7 \%)$ & $0(0 \%)$ & $1(4,3 \%)$ & $0(0 \%)$ \\
\hline $\begin{array}{l}\text { Tengo que cuidar a } \\
\text { familiares mayores }\end{array}$ & $0,39 \pm 0,94$ & $19(82,6 \%)$ & $1(4,3 \%)$ & $1(4,3 \%)$ & $2(8,7 \%)$ \\
\hline Tengo una discapacidad & $0,13 \pm 0,46$ & $21(91,3 \%)$ & $1(4,3 \%)$ & $1(4,3 \%)$ & $0(0 \%)$ \\
\hline
\end{tabular}


Tabla 3. Resultados de los motivos percibidos por los participantes para la práctica de actividad física.

\begin{tabular}{|c|c|c|c|c|c|}
\hline Ítem/pregunta & Media $\pm D T$ & Nada & Algo & Bastante & Mucho \\
\hline $\begin{array}{l}\text { Para recuperarme de una } \\
\text { enfermedad o lesión }\end{array}$ & $0,35 \pm 0,93$ & $20(87 \%)$ & $0(0 \%)$ & $1(4,3 \%)$ & $2(8,7 \%)$ \\
\hline Para controlar el peso & $1,48 \pm 1,20$ & $7(30,4 \%)$ & $2(17,4 \%)$ & $6(26,1 \%)$ & $6(26,1 \%)$ \\
\hline $\begin{array}{l}\text { Para estar en forma } \\
\text { Porque haciendo actividad }\end{array}$ & $2,26 \pm 1,01$ & $2(8,7 \%)$ & $3(13 \%)$ & $5(56,5 \%)$ & $13(56,5 \%)$ \\
\hline $\begin{array}{l}\text { física me siento bien } \\
\text { conmigo mism@ }\end{array}$ & $2,87 \pm 0,46$ & $0(0 \%)$ & $1(4,3 \%)$ & $1(4,3 \%)$ & $21(91,3 \%)$ \\
\hline $\begin{array}{l}\text { Porque es un tiempo solo } \\
\text { para mi }\end{array}$ & $2,48 \pm 1,04$ & $3(13 \%)$ & $0(0 \%)$ & $3(13 \%)$ & $17(73,9 \%)$ \\
\hline $\begin{array}{l}\text { Para encontrarme con mis } \\
\text { amistades }\end{array}$ & $2,57 \pm 0,94$ & $2(8,7 \%)$ & $1(4,3 \%)$ & $2(8,7 \%)$ & $18(78,3 \%)$ \\
\hline $\begin{array}{l}\text { Por diversión } \\
\text { Porque disfruto aprendiendo }\end{array}$ & $2,91 \pm 0,29$ & $0(0 \%)$ & $0(0 \%)$ & $2(8,7 \%)$ & $21(91,3 \%)$ \\
\hline cosas nuevas & $2,83 \pm 0,49$ & $0(0 \%)$ & $1(4,3 \%)$ & $2(8,7 \%)$ & $20(87 \%)$ \\
\hline Para mejorar mi salud física & $2,61 \pm 0,66$ & $0(0 \%)$ & $2(8,7 \%)$ & $5(56,5 \%)$ & $16(69,6 \%)$ \\
\hline $\begin{array}{l}\text { Para mejorar mi bienestar } \\
\text { mental }\end{array}$ & $2,43 \pm 0,79$ & $0(0 \%)$ & $4(17,4 \%)$ & $5(21,7 \%)$ & $14(60,9 \%)$ \\
\hline $\begin{array}{l}\text { Porque me gusta hacer esta } \\
\text { actividad física }\end{array}$ & $2,87 \pm 0,34$ & $0(0 \%)$ & $0(0 \%)$ & $3(13 \%)$ & $20(87 \%)$ \\
\hline $\begin{array}{l}\text { Para eliminar el estrés } \\
\text { Para mejorar mi estado de }\end{array}$ & $2,04 \pm 1,11$ & $3(13 \%)$ & $4(17,4 \%)$ & $5(21,7 \%)$ & $11(47,8 \%)$ \\
\hline animo & $2,39 \pm 0,89$ & $1(4,3 \%)$ & $3(13 \%)$ & $5(21,7 \%)$ & $14(60,9 \%)$ \\
\hline $\begin{array}{l}\text { Para relacionarme con otras } \\
\text { personas }\end{array}$ & $2,61 \pm 0,94$ & $2(8,7 \%)$ & $1(4,3 \%)$ & $1(4,3 \%)$ & $19(82,6 \%)$ \\
\hline $\begin{array}{l}\text { Para pasar más tiempo con } \\
\text { mi pareja }\end{array}$ & $0,52 \pm 0,90$ & $18(78,3 \%)$ & $1(4,3 \%)$ & $1(4,3 \%)$ & $3(13 \%)$ \\
\hline $\begin{array}{l}\text { Porque me siento mal si no } \\
\text { practico } \\
\text { Porque me gusta superar }\end{array}$ & $1,00 \pm 1,09$ & $10(43,5 \%)$ & $6(26,1 \%)$ & $4(17,4 \%)$ & $3(13 \%)$ \\
\hline retos & $2,61 \pm 0,89$ & $2(8,7 \%)$ & $0(0 \%)$ & $3(13 \%)$ & $18(78,3)$ \\
\hline $\begin{array}{l}\text { Porque quiero que forme } \\
\text { parte de mi estilo de vida }\end{array}$ & $2,52 \pm 0,90$ & $2(8,7 \%)$ & $0(0 \%)$ & $5(21,7 \%)$ & $16(69,6 \%)$ \\
\hline $\begin{array}{l}\text { Por las sensaciones positivas } \\
\text { que me produce realizar AF }\end{array}$ & $2,70 \pm 0,70$ & $0(0 \%)$ & $3(13 \%)$ & $1(4,3 \%)$ & $19(82,6 \%)$ \\
\hline $\begin{array}{l}\text { Porque me permite estar en } \\
\text { la naturaleza }\end{array}$ & $2,48 \pm 0,79$ & $1(4,3 \%)$ & $1(4,3 \%)$ & $7(30,4 \%)$ & $14(60,9 \%)$ \\
\hline \multirow{2}{*}{$\begin{array}{l}\text { Por obligación } \\
\text { Por recomendación medica }\end{array}$} & $0,26 \pm 0,69$ & $19(82,6 \%)$ & $3(13 \%)$ & $0(0 \%)$ & $1(4,3 \%)$ \\
\hline & $0,78 \pm 1,13$ & $14(60,9 \%)$ & $3(13 \%)$ & $3(13 \%)$ & $3(13 \%)$ \\
\hline $\begin{array}{l}\text { Para mejorar mi aspecto } \\
\text { físico }\end{array}$ & $1,83 \pm 1,19$ & $4(17,4 \%)$ & $6(26,1 \%)$ & $3(13 \%)$ & $10(43,5 \%)$ \\
\hline $\begin{array}{l}\text { Para mejorar mi capacidad } \\
\text { física }\end{array}$ & $2,39 \pm 0,99$ & $2(8,7 \%)$ & $2(8,7 \%)$ & $4(17,4 \%)$ & $15(65,2 \%)$ \\
\hline $\begin{array}{l}\text { Para desconectar un rato } \\
\text { Para compartir actividades }\end{array}$ & $2,65 \pm 0,71$ & $1(4,3 \%)$ & $0(0 \%)$ & $5(21,7 \%)$ & $17(73,9 \%)$ \\
\hline con mis hij@s & $0,13 \pm 0,63$ & $22(95,7 \%)$ & $0(0 \%)$ & $0(0 \%)$ & $1(4,3 \%)$ \\
\hline
\end{tabular}


muestran que las barreras más destacadas fueron "no me siento a gusto con mi cuerpo" y "me da pereza". Respecto a los facilitadores, los mejor puntuados fueron "por diversión" y "porque haciendo actividad física me siento bien con-migo mism@". Los resultados del presente estudio pueden ser relevantes para conocer cuáles son las barreras y motivos de practica de AF de personas con discapacidad que asiduamente practican alguna modalidad deportiva y en con-secuencia poder orientar la oferta de AF en función de las dificultades y motivos percibidos por personas con discapacidad.

El análisis de las barreras percibidas por personas con discapacidad ya ha sido analizado en anteriores estudios ${ }^{23,25}$. Se ha expuesto que es de gran importancia conocer la percepción de las barreras que tienen las personas con discapacidad para la práctica de AF, ya que esto puede permitir actuar sobre estas barreras con el objetivo de poder modificarlas ${ }^{26}$. Los resultados obtenidos en el presente estudio muestran que las barreras más relevantes para la práctica de AF en personas con discapacidad fueron la pereza y el no sentirse a gusto con su cuerpo. Por el contrario, la puntuación más baja se encontró en los ítems "no me siento capaz" $(0,00 \pm 0,00)$ y "los técnicos no son adecuados" $(0,00 \pm 0,00)$. Estos resultados concuerdan con estudios anteriores donde también se ha descrito que la pereza era una de las barreras a la hora de realizar AF en jóvenes con PC23 y que no sentirse a gusto con su cuerpo también fue otra de las grandes barreras en adolescentes con PC25. Sin embargo, otros estudios apuntan distintas barreras percibidas por personas con discapacidad. Úbeda-Colomer et al. ${ }^{27}$ en un estudio realizado con alumnos universitarios con discapacidad, observaron que una de las barreras más importantes fue el hecho de sentirse incapaz de practicar AF. Por otro lado, la falta de adecuación de los monitores o entrenadores, fue una de las barreras en otro estudio realizado por Brian y Haegele ${ }^{28}$. En nuestro estudio, ni el no sentirse capaz ni la falta de adecuación de los monitores o entrenadores fueron percibidas como barrera principal percibida por los deportistas. Cabe destacar que en el presente estudio ninguna barrera alcanzo puntuaciones altas y que los porcentajes de "nada" en todos los ítems de barreras fueron muy elevados. Estas diferencias entre estudios y especialmente la baja percepción de la existencia de barreras declarada por los deportistas participantes en nuestro estudio puede estar condicionada por las características de la muestra. En nuestro estudio participaron personas con discapacidad asiduamente practicantes de un deporte de equipo, con muchos años de experiencia y en un entorno deportivo controlado, aspecto que ha podido condicionar que la percepción de las barreras sea menor.

En la literatura, además de las barreras, también se han analizado los aspectos facilitadores para la práctica de AF en personas con discapacidad ${ }^{29}$. Al igual que en el caso de las barreras, conocer cuáles son los facilitadores de práctica de AF puede ayudar a conocer lo que desean los participantes y poder ofertar un tipo de AF más acorde a sus preferencias. Los resultados obtenidos en el presente estudio muestran que la media de las respuestas fue superior a 2 puntos en muchos de los ítems, como por ejemplo en los ítems "para encontrarme con mis amistades", "para estar en forma" y "para mejorar mi bienestar mental". 
Las preguntas que recibieron mayor puntuación fueron "realizar AF por diversión" y "para sentirse bien con uno mism@". Sin embargo, los ítems "por obligación" $(0,26 \pm 0,69)$, "para compartir actividades con mis hij@s" $(0,13$ $\pm 0,63)$ y "para recuperarme de una enfermedad o lesión" $(0,35 \pm 0,93)$ fueron los menos puntuados. Estos resultados coinciden con los resultados obtenidos en otro estudio realizado por Shields y Synnot ${ }^{29}$ donde los autores analizaron la percepción de niños con discapacidad, sus padres y sus técnicos deportivos, y se observó que la diversión era uno de los mayores facilitadores para la práctica de AF. Otros estudios también han observado que el apoyo o el encuentro con sus amistades ${ }^{22,26}$, el bienestar mental ${ }^{22,30}$ y el estar en forma o saludable 22,31 son grandes motivos para la práctica de AF. Atendiendo a los resultados obtenidos, parece que algunas de las características que debe tener la práctica de AF para personas con discapacidad debe ser la práctica en grupo, donde se favorezca el ámbito social, lúdico y orientado a la salud y el bienestar.

Contrariamente a los resultados obtenidos en otras investigaciones anteriores, en el presente estudio los deportistas con discapacidad participantes no puntuaron de forma alta las barreras. Este aspecto puede deberse al hecho de que eran deportistas con alta experiencia y practicaban AF de forma habitual, aspecto que ha podido hacer que muchas de las barreras ya estén superadas. Con respecto a los motivos de práctica de AF más destacados fueron "por diversión" y "porque haciendo actividad física me siento bien conmigo mism@". Estos resultados ponen de manifiesto que la práctica de AF debería ir especialmente orientada a tareas en grupo, lúdicas y que persigan un objetivo de salud y de bienestar.

\section{LIMITACIONES Y CAMINOS FUTUROS}

El presente estudio no está exento de limitaciones. Cabe destacar que la muestra (tamaño y características) no es representativo de las personas con discapacidad, por lo que no es posible generalizar los resultados. Además, todos los participantes proceden de un mismo entorno deportivo, y el hecho de ser deportistas con experiencia puede condicionar los resultados obtenidos. Por lo tanto, sería interesante en futuros estudios ampliar la muestra de estudio para que sea más representativa de la población, así como analizar qué oferta de AF prefieren las personas con discapacidad para poder colaborar en la mejora del nivel de práctica de AF en personas con discapacidad.

\section{REFERENCIAS BIBLIOGRÁFICAS}

1. Wilson MG, Ellison GM, Cable NT. Basic science behind the cardiovascular benefits of exercise. Br J Sports Med. 2016;50(2):93-99. https://doi.org/10.1136/bjsports2014-306596rep

2. Rhodes RE, Janssen I, Bredin SSD, Warburton DER, Bauman A. Physical activity: Health impact, prevalence, correlates and interventions. Psychol Health. 2017;32(8):942-975. https://doi.org/10.1080/08870446.2017.1325486 
3. Anziska Y, Inan S. Exercise in neuromuscular disease. Semin Neurol. 2014;34(5):542556. https://doi.org/10.1055/s-0034-1396008

4. Organización mundial de la salud (OMS). monitoring health for the SDGs, sustainable development goals. Geneva: World Health Organization; 2019.

5. Warburton DER, Nicol CW, Bredin SSD. Health benefits of physical activity: the evidence. CMAJ. 2006;174(6):801-809. https://doi.org/10.1503/cmaj.051351

6. Rimmer JH, Braddock D, Pitetti KH. Research on physical activity and disability: An emerging national priority. Med Sci Sports Exerc. 1996;28(11):1366-1372. https:// doi.org/10.1097/00005768-199611000-00004

7. Heller T, McCubbin JA, Drum C, Peterson J. Physical activity and nutrition health promotion interventions: What is working for people with intellectual disabilities? Intellect Dev Disabil. 2011;49(1):26-36. https://doi.org/10.1352/1934-9556-49.1.26

8. Collins K, Staples K. The role of physical activity in improving physical fitness in children with intellectual and developmental disabilities. Res Dev Disabil. 2017;69:49_ 60. https://doi.org/10.1016/j.ridd.2017.07.020

9. Van Den Berg-Emons R, Van Baak M, Speth L, Saris W. Physical training of school children with spastic cerebral palsy: Effects on daily activity, fat mass and fitness. Int J Rehabil Res. 1998;21(2):179-194.

10. Martin JJ. Benefits and barriers to physical activity for individuals with disabilities: A social-relational model of disability perspective. Disabil Rehabil. 2013;35(24),20302037. https://doi.org/10.3109/09638288.2013.802377

11. Kristén L, Patriksson G, Fridlund B. Conceptions of Children and Adolescents with Physical Disabilities about Their Participation in a Sports Programme. Eur Phy Educ Rev. 2002;8(2):139-156. https://doi.org/10.1177/1356336X020082003

12. Badia M, Longo E. El ocio en las personas con discapacidad intelectual: participación y calidad de vida a través de las actividades de ocio. Revista Española Sobre Discapacidad Intelectual. 2009;40(3):30-44.

13. Martin JJ. Psychosocial aspects of youth disability sport. Adapt Phys Acti Q. 2006;23:65-77.

14. Anderson DM, Wozencroft A, Bedini LA. Adolescent Girls' Involvement in Disability Sport: A Comparison of Social Support Mechanisms. J Leis Res. 2008;40(2):183207. https://doi.org/10.1080/00222216.2008.11950137

15. McKeon M, Slevin E, Taggart L. A pilot survey of physical activity in men with an intellectual disability. J Intellect Disabil. 2013;17(2):157-167. https://doi. org/10.1177/1744629513484666

16. Wouters M, Evenhuis HM, Hilgenkamp TIM. Physical activity levels of children and adolescents with moderate-to-severe intellectual disability. J Appl Res Intellect Disabil. 2018;32(1):131-142. https://doi.org/10.1111/jar.12515

17. Tollerz LUB, Forslund AH, Olsson RM, Lidström H, Holmbäck, U. Children with cerebral palsy do not achieve healthy physical activity levels. Acta Paediatrica. 2015;104(11):1125-1129. https://doi.org/10.1111/apa.13141 LK

18. Gallego J, Aguilar-Parra JM, Cangas, JA, Pérez-Escobar, JM, \& Barrera, S. Hábitos de actividad física en mujeres con discapacidad: relación con sus características físicas y funcionales. Revista Iberoamericana de Psicología del Ejercicio y el Deporte. 2014;9(2):471-494. 
19. Draheim CC, Williams DP, McCubbin JA. Prevalence of Physical Inactivity and Recommended Physical Activity in Community-Based Adults With Mental Retardation. Ment. Retard. 2002;40(6):436-444. https:// doi.org/10.1352/0047-6765(2002)040<0436: popiar>2.0.co;2

20. National Center for Health. Healty People 2010 Final Review. Hyattsville: DHHS publication; 2012.

21. Malone LA, Barfield JP, Brasher JD. Perceived benefits and barriers to exercise among persons with physical disabilities or chronic health conditions within action or maintenance stages of exercise. Disabil Health J. 2012;5(4):254-260. https://doi. org/10.1016/j.dhjo.2012.05.004

22. Abellán J, Januário N. Barreras, facilitadores y motivos de la práctica deportiva de deportistas con discapacidad intelectual. Psychol, society and Educ. 2017;9(3):419-431. https://doi.org/10.25115/psye.v9i3.1023

23. Verschuren O, Wiart L, Hermans D, Ketelaar M. Identification of facilitators and barriers to physical activity in children and adolescents with cerebral palsy. J Pediatr. 2012;161(3):488-494. https://doi.org/10.1016/j.jpeds.2012.02.042

24. Kostiuk LV. Barreras para la práctica de actividad física y deportiva en las personas adultas de la Comunidad de Madrid. Desarrollo y validación de un instrumento (tesis doctoral). Madrid: Universidad Politécnica de Madrid; 2016. 109 p. https://doi. org/10.20868/UPM.thesis.39693.

25. Conchar L, Bantjes J, Swartz L, Derman W. Barriers and facilitators to participation in physical activity: The experiences of a group of South African adolescents with cerebral palsy. J Health Psychol. 2014;21(2):152-163. https://doi. org/10.1177/1359105314523305

26. Jaarsma EA, Geertzen JHB, de Jong R, Dijkstra PU, Dekker R. Barriers and facilitators of sports in Dutch Paralympic athletes: An explorative study. Scand J Med Sci Sports. 2014;24(5):830-836. https://doi.org/10.1111/sms.12071

27. Úbeda-Colomer J, Molina-Alventosa P, Campos-Granell J. Facilitadores y barreras para la actividad física en tiempo de ocio en alumnado universitario con discapacidad: un estudio cualitativo. Educación Física y Deporte. 2016;35(1):63-96. http://doi. org/10.17533/udea.efyd.v35n1a03

28. Brian A, Haegele JA. Including students with isual Impairments: Softball. J Phys Educ, Recreat Dance. 2014;85(3):39-45. http:// doi.org/10.1080/07303084.2014.984733

29. Shields N, Synnot A. Perceived barriers and facilitators to participation in physical activity for children with disability: A qualitative study. BMC Pediatrics. 2016;16(1):1-10. https://doi.org/10.1186/s12887-016-0544-7

30. Úbeda-Colomer J, Monforte J, Campos-Granell J, Llopis-Goig R, Torregrosa-Cabrera MÁ, Devís-Devís J. Motivos de práctica y abandono físico-deportivo en alumnado universitario con discapacidad. Cultura, Ciencia y Deporte. 2018;12(37):51-60.

31. Lomas A, Clemente AL. Beneficios de la actividad físico-deportiva en niños y niñas con TDAH. EmásF: revista digital de educación física. 2017;44:63-78. 